Originalveröffentlichung in: Grenzmann, Ludger; Haye, Thomas ; Henkel, Nikolaus ; Kaufmann, Thomas (Hrsgg.): Wechselseitige Wahrnehmung der Religionen im Spätmittelalter und in der Frühen Neuzeit, Teil 1: Konzeptionelle Grundfragen und Fallstudien, Berlin, New York 2009, S. 73-99 (Abhandlungen der Akademie der Wissenschaften zu Göttingen ; N. F. Bd. 4)

\title{
Margaretes Paradiesvogel. \\ Vereinnahmungen des Fremden und Wunderbaren aus der Neuen Welt im frühneuzeitlichen Kunstdiskurs
}

\section{Christian Freigang}

Mit der Entdeckung der Neuen Welt vollzog sich ein epochaler Wandel in der europäischen Wissens- und Wahrnehmungskultur. Wenn Kolumbus glaubte, den Ostteil Asiens entdeckt zu haben, bedeutete dies, ein phantastisches Land betreten zu haben, das bislang nur in mythischen Berichten existierte. Und als sich zunehmend die Erkenntnis durchsetzte, dass hier ein neuer Kontinent gefunden worden war, stellte dies bekanntlich grundsätzlich neue Fragen, etwa zum göttlichen Schöpfungsplan oder der menschlichen Natur der Ureinwohner. Die Deskriptionen des Neuen bilden allein aufgrund der Vielfalt der Entdeckungen und der umfangreichen topischen und literarischen Überformungen ein äußerst heterogenes Bild, das zwischen dem gleichsam im Paradies lebenden edlen Wilden und dem unmenschlichen und sittenlosen Kannibalen oszilliert. Die Naturalien und Artefakte, die mit den Entdeckungsfahrten nach Europa gelangten, spielten dabei eine nicht unwichtige Rolle. Allerdings fällt auf, dass das Interesse an den Stücken aus Übersee sich nicht auf ihre Qualität als $\mathrm{Na}$ turalie, als Exoticum, als Trophäe oder als ethnographisches Dokument beschränkte. Ihre epistemologische Verortung war vielmehr auch Teil einer weitreichenden, sich damals in der Alten Welt im Wandel befindlichen Auffassung von künstlerischer Produktion und Wahrnehmung. Kunst als Wissenssystem erst ermöglichte die klassifizierende Aneignung des Fremden. Diesem Aspekt sind die folgenden Ausführungen gewidmet, wobei sich der zeitliche Rahmen im wesentlichen auf das erste Drittel des 16 . Jahrhunderts beschränken muss. ${ }^{1}$

1 Als allg. Literatur zur frühen Rezeption der Neuen Welt in Europa sei auswahlweise genannt: Benjamin Keen: The Aztec Image in Western Thought. New Brunswick 1971; Hugh Honour: The New Golden Land. European Images of America from the Discoveries to the Present Time. London 1975; Wolfgang Reinhard (Hrsg.): Humanismus und Neue Welt. Weinheim 1987 (=Mitteilung XV der Kommission für Humanismusforschung); Wolfgang Neuber: Fremde Welt im europäischen Horizont: zur Topik der deutschen Amerika-Reiseberichte der Frühen Neuzeit. Berlin 1991 (= Philologische Studien und Quellen, H. 121); Urs BitTerli/Eberhard SchmitT (Hrsg.): Die Kenntnis beider „Indien“ im frühneuzeitlichen Europa. München 1991 (=Akten der Zweiten Sektion des 37. deutschen Historikertages in Bamberg 1988); Paul Vandenbroeck (Hrsg.): America : Bride of the sun ; 500 years Latin America and the Low Countries. Ausst. Kat. Antwerpen 1991. Gent 1991; Peter Mesenhöller 
In einem ersten Teil wird es um die Bewertung des Paradiesvogels als Preziose göttlicher Schöpfung gehen, der zweite Abschnitt fokussiert auf die Bewertung der künstlerischen Produktion aus der Neuen Welt, der dritte schliesslich behandelt die kunsttheoretische Bedeutung von Phantasie bei der Wahrnehmung des fremden Kontinents.

In ihrer Residenz in Mecheln verwahrte Margarete von Österreich (14801530) - Tochter von Kaiser Maximilian und Tante Kaiser Karls V. - seit den ersten Jahren des 16. Jahrhunderts eine umfangreiche Kunst-, Naturalien- und Büchersammlung. Diese zählte, wie zuletzt von Dagmar Eichberger eingehend dargestellt, zu den ersten und zugleich wichtigsten Beispielen einer systematisch, d.h. vor allem nach genealogischen, religiösen und künstlerischen Kriterien geordneten fürstlichen Kollektion. ${ }^{2}$ Neben einer Vielzahl von Portraits, reli-

(Hrsg.): Mundus Novus. Amerika oder die Entdeckung des Bekannten. Das Bild der Neuen Welt im Spiegel der Druckmedien vom 16. bis zum frühen 20. Jahrhundert. Essen 1992; Hans Wolff (Hrsg.): America. Das frühe Bild der Neuen Welt. Ausst. Kat. München 1992. München 1992; Jay A. Levenson (Hrsg.): Circa 1492. Art in the Age of Exploration. Ausst. Kat. Washington 1992. New Haven u.a. 1992; Hildegard FrüвIs: Die Wirklichkeit des Fremden. Die Darstellung der Neuen Welt im 16. Jh. Berlin 1995; Mary Louise Krumrine/Susan Clare Scott (Hrsg.): Art and the Native American Perceptions, Reality, and Influences. University Park (Penn.) 2001 (= Papers in Art History from The Pennsylvania State University, vol. X); Renate Pieper: Die Vermittlung der Neuen Welt. Amerika im Nachrichtennetz des habsburgischen Imperiums 1493-1598. Mainz 2000 (=Veröffentlichungen des Instituts für europäische Geschichte Mainz, Abt. Universalgeschichte, Bd. 163). - Grundlegend zum Thema der Rezeption der Produkte aus der Neuen Welt sind die Beiträge von Christian F. Feest: Vienna's Mexican Treasures. Aztec, Mixtec, and Tarascan Works from 16th Century Austrian Collections, in: Archiv für Völkerkunde 44/1990, S. 1-63; Christian F. Feest: „Selzam ding von gold da von vill zu schreiben were“: Bewertungen amerikanischer Handwerkskunst im Europa des frühen 16. Jahrhunderts, in: Stephan Füssel (Hrsg.): Die Folgen der Entdeckungsreisen für Europa. Akten des interdisziplinären Symposions 12./13. April in Nürnberg. Nürnberg 1992 (= Pirckheimer-Jahrbuch 7/1992); Christian F. FeEst: European Collection of American Indian Artefacts and Art, in: Journal of the History of Collections 5/1993, S. 1-11.

2 Dagmar Erchberger: Leben mit Kunst, Wirken durch Kunst. Sammelwesen und Hofkunst unter Margarete von Österreich, Regentin der Niederlande. Turnhout (= Burgundica, V); Eichberger, Dagmar: Devotional Objects in Book Format : Diptychs in the Collection of Margaret of Austria and her Familiy, in: Margaret M. Manion / Bernard J. Muir (Hrsg.): The Art of the Book. Its Place in Medieval Worship. Exeter 1998, 291-323; Dagmar EichBerger: Margaret of Austria's portrait collection : female patronage in the light of dynastic ambitions and artistic quality, in : Renaissance Studies 10/1996, S. 259-279; Dagmar Eichberger / Lisa Beaven: Family Members and Political Allies : The Portrait Collection on Margaret of Austria, in : Art Bulletin 77/1993, S. 225-248; Joris Capenberghs: Margaret of Austria, the Hof van Savoyen and the New World, in: Dagmar Eichberger (Hrsg.) : Women of Distinction. Margaret of York, Margaret of Austria. Ausst. Kat. Mechelen 2005. Davidsfonds/Leuven 2005, S. 296-309. 
giösen Bildern und exotischen Naturalia fanden sich hier auch eine Reihe von Preziosen aus der Neuen Welt: Bekleidungsstücke, Schmuckstücke und insbesondere farbenprächtige Federschmucke, welche das Inventar von 1523 unter dem Titel Accoustrement de plumes venuz des Indes verzeichnet. Die Sammlung war als Geschenk Karls V. nach Mecheln gekommen und wurde hier geschlossen in der Bibliothek verwahrt. ${ }^{3}$ Das kostbarste Objekt unter den Stücken aus Übersee war indessen kein Artefakt, sondern ein Naturprodukt, nämlich der in einem hölzernen Schächtelchen verwahrte und mit Taft eingeschlagene Balg eines Paradiesvogels: Item, ung oyseau mort, appellé oyseau de paradis, envelopé de taffetaf, mis en ung petit coffret de bois. Er befand sich, zusammen mit vielen anderen Kunstgegenständen in dem sogenannten Kleinen Kabinett, dem privaten Studiolo der Fürstin. ${ }^{4}$

Charakteristika des Vogels sind zwar weiter nicht angegeben, doch mit guten Gründen ist zu vermuten, dass es sich bei dem oyseau de paradis tatsächlich um ein Exemplar der Spezies der Paradiesvögel handelte: Die Nennung im Inventar der Herrscherin meint ganz offensichtlich einen fremden exotischen Vogel, und nicht etwa einfach einen Papagei oder Sittich. Der Inventarisator, dem es ja auf die präzise Identifizierbarkeit der Vögel ankam, hatte alles Interesse, hier eine möglichst genaue Benennung anzugeben. Und so haben wir allen Anlass anzunehmen, dass Margaretes Paradiesvogel entweder ein südamerikanischer Ara oder einer jener ersten fünf nach Europa gelangten Paradiesvögel aus der Südsee war. 1521 waren diese als Geschenk der Bevölkerung der Molukken der Mannschaft Magellans bei deren Weltumsegelung übergeben worden. Einer der Vogelbälge wurde durch den kaiserlichen Sekretär, Maximilian von Transsylvanien, Kaiser Karl V. überbracht und dabei als ursprünglich aus dem Paradies kommend bezeichnet. ${ }^{5}$ Weiterhin wissen wir, dass ein Teil der Geschenke, die der Aztekenherrscher Moctezuma vor seiner Beseitigung durch Hernan Cortés dem Kaiser überreichen ließ, von diesem an seine Tante weiterverteilt wurde. Möglich ist solche eine Weitergabe auch im Fall des Paradiesvogels. ${ }^{6}$

3 M. Michelant: Inventaire des vaisselles, joyaux, tapisseries, peintures, manuscrits, etc., de Marguerite d'Autriche, régente et gouvernante des Pays-Bas, dressé en son palais de Malines, le 9 juillet 1523, in : Comte rendu des séances de la Commission royale d'histoire, ou recueil de ses bulletins, $3^{\text {e }}$ sér., 12/1871, S. 5-78 u. 83-136, hier S. $61-65$.

4 Michelant (Anm. 3), S. 96, s. a. Eichberger, Leben mit Kunst (Anm. 2), S. 185 u. $372-388$.

5 Erwin Stresemann: Die Entdeckungsgeschichte der Paradiesvögel, in: Journal für Ornithologie 95/1954, S. 263-291; Georg Horstrus: Gesneri Redivivi, aucti \& emendati tomus II. Oder Vollkommenes Vogel=Buch ... Frankfurt 1669, S. 363. Zu Maximilian von Transsylvaniens Bericht s. u.

6 Allerdings ist einzuwenden, dass die Geschenke geschlossen in der Bibliothek in Mecheln verwahrt wurden, ausgerechnet der Paradiesvogel sich indessen im Studiolo befand. 
Hinter dem farbenprächtige Vogel aus der Fremde lagen vielschichtige Bedeutungsdimensionen und Konnotationen. Das gilt schon allein für die bemerkenswert frühe Verwendung seiner auch heute noch geläufigen begrifflichen Bezeichnung: Paradiesvogel. Denn damit war zunächst zu assoziieren, dass nunmehr dank der Entdeckungsreisen nach Westindien das irdische Paradies am Rande der Welt, mitsamt seiner wunderbaren Fauna und Flora gefunden war. Ähnliche Rezeptionsmuster waren kurz zuvor im Fall der amerikanischen Papageien wirksam geworden, die die mythischen Beschreibungen Indiens mit seiner farbenprächtigen Wunderwelt nun über Realien markant zu bestätigten schienen. Bekanntlich wurde der Papagei umgehend zum Symbol der Neuen Welt und ihrer verwunderlichen Fremdheit - auf der ersten Waldseemüllerkarte von 1507 erscheint er emblemhaft für Amerika und in der Carta Marina von 1516 heisst es: Prisilia sive terra papagalli: Ein Tier, das in Europa Fürsten vorbehalten war, diente in der Karibik angeblich der Bevölkerung als Nahrung. ${ }^{7}$ Im Fall des Paradiesvogels sind noch andere Momente wirksam. Für Maximilian van Transsylvanien ist der Vogel das spektakulärste, kostbarste Geschenk aus der Südsee. Anlässlich der Weiterleitung an Karl V. hebt er hervor, dass der Balg nicht deswegen übergeben werde, damit nun auch der Kaiser dem Aberglauben der Eingeborenen anhinge, der Besitz des Vogels verleihe Unsterblichkeit, sondern damit er sich an seiner Seltenheit und Pracht ergötze. Und weiter heißt es in dem 1523 auch gedruckten Schreiben, dass die Inselbewohner glaubten, der Vogel würde die Seele unsterblich machen, und dies liege daran, dass er niemals Erdkontakt habe. Moslemische Händler hätten den Molukkern versichert, der Vogel komme aus dem Paradies, wo sich die Seelen der Toten aufhielten. Deswegen sei die Urbevölkerung zum Islam konvertiert und habe den Vogel Mamuco Codiata, Gottesvogel, genannt. ${ }^{8}$ Diese ornithologischen Erläuterungen finden sich in Variation auch in Antonio Pigafettas Reisebeschreibung Magellans zu den Molukkeninseln. Der drosselgroße, außergewöhnlich schöne Vogel habe keine Flügel, stattdessen nur Federn in verschiedensten Farben. Er fliege nicht selbst, sondern treibe im Wind. Nach Auffassung der Einheimischen komme er aus dem irdischen Paradies und werde deswegen Gottesvogel genannt. ${ }^{9}$ In Anlehnung an Maximilian von Transsylvanien weiß auch der Ornithologe Konrad Gesner in seinem 1555 erschienenen Vogelkompendium weiter zu berichten, dass die Paradiesvögel deswegen keine Füße hätten, weil sie

7 Pieper (Anm. 1), S. 248-249.

8 Maximilianus Transsylvanus: Epistola de moluccis insulis ... Köln 1523; Stresemann (wie Anm. 5), s. a. Charles E. Nowell (Hrsg.): Antonio Pigafetta, Maximilian of Transsylvania, Gaspar Corrêa: Magellan's Voyage around the World. Three Contemporary Accounts. Evanston 1962, S. 303 u. 307.

9 Antonio Pigafetta: Le voyage et navigation ... en Isles des Mollucques 1524-34, zit. nach Stresemann (wie Anm. 5); Nowell (Anm. 8), S. 222 
sich beständig in der Luft aufhielten und sich dort vom Tau des Himmels ernährten. ${ }^{10}$ Diese Annahmen gehen offensichtlich zurück auf Plutarchs Äußerungen zu der mythischen Gattung der Rhyntakes in Persien, die aufgrund der genannten Ernäherungsweise frei von Exkrementen und lediglich mit Fett gefüllt seien. ${ }^{11}$ Der französische Ornithologe Belon identifiziert 1555 die Ryntakes mit einem anderen mythischen farbenprächtigen Vogel, dem Phoenix. ${ }^{12}$ Aus dieser Kompilation ornithologischen Vorwissen erklärt es sich offenbar, dass schon in den dreißiger Jahren des 16. Jahrhunderts die einheimischen Paradiesvogeljäger den Bälgen die Füße und bisweilen auch die Flügel abschnitten. Damit sahen die Tiere tatsächlich ihrer mythischen Beschreibung gemäß aus, und ihre Federpracht kam noch intensiver zum Vorschein. In dieser Gestalt finden wir die ersten Abbildungen von Paradiesvögeln bereits seit ca. 1525 in einer unscheinbar wirkenden Silberstiftzeichnung von Hans Baldung Grien und weiterhin in einer Reihe farbenfroher Aquarelle der zweiten Hälfte des 16. Jahrhunderts. Auffällig ist die fast abstrakte Qualität dieser Bilder, die eher als Farbstudie denn als getreue ornithologische Notierung zu gelten haben. ${ }^{13}$

Außer den Paradiesvögeln der Südsee benennt Gesner in seiner „Historia Animalium" weitere Arten, die Paradiesvögel genannt werden, und zwar wegen der Buntheit ihrer Federn: Sie halten sich über dem Fluß Nilo auff, welcher aus dem Paradiß fliessen soll, sonst werden sie selten gefunden. Als wir im Fluß Physon geschiffet (sagt einer, welcher die Histori von dem gelobten Land Italianisch beschrieben hat) haben wir Vögel gefunden, welche Paradißsögel genennet werden, mit wunderschönen Farben gezieret, daß wenn einer sie eigentlich anschauet, verblenden sie einem das Gesicht. ${ }^{14}$ Diese Information gehen nicht auf die neueren Erkenntnissen der Forschungsreisen, sondern auf die mittelalterlichen Enzyklopädisten wie Vinzenz von Beauvais und Albertus Magnus zurück. Letzter gibt unter dem Eintrag De Avibus paradisi an: quae paradisi dicuntur ob decorem: quia nullus eis decor videtur deesse. ${ }^{15}$ Und im "Speculum naturale" des Vinzenz

10 Conrad Gesner: Historiae animalium Liber III. qui est de avium natura. Zürich 1555 , S. 611-614; Horstium (wie Anm. 5).

11 Plutarch, Vita Artaxerxis, 19 (Plutarch's Lives, Bd. 11, London u. Cambridge (Mass.) 1962, S. 170).

12 Pierre Belon: Histoire de la nature des Oyseaux. Paris 1555, S. 330.

13 Fritz Koreny: Albrecht Dürer und die Tier- und Pflanzenstudien der Renaissance. München 1985, Nr. 30-34.

14 Horstium (wie Anm. 5), S. 364.

15 Albertus Magnus: Opera Omnia, Bd. XII, Paris 1891 : De Animalibus, Lib. XXIII, Tract. I, Nr. 12: De Avibus paradisi (S. 444): Aves paradisi vocant Aegyptii quasdam aves magnitutinis anserum, quae paradisi dicuntur ob decorem : quia nullus eis decor videtur deesse, et hae aves illaqueatae gemere non cessant donec moriantur vel donatae fuerint libertati. Liberae autem adeo dulciter modulantur, quod in omni audiente delectationem inducunt. Harum habitatio est super Nilum qui de paradiso fluere perhibetur. 
von Beauvais heißt es noch weiter präzisierend: ... aves paradisi vulgariter sic appellantur, non quia de paradiso sint, sed ob insignem pulchritudinem: tanti namque sunt decoris, \& gloriae, ut nullus eis color credatur deesse $\ldots{ }^{16}$ Hier gehen wiederum Topoi ein, die aus den antiken Beschreibungen des Phoenix entnommen sind: Gemäß Plinius lebe dieser in Arabien, sei so groß wie ein Adler, goldglänzend um den Hals, ansonsten purpurfarben, rosafarbene Federn modulierten den bläulichen Schwanz. Die Kehle sei geschmückt mit Auswüchsen und der Kopf mit einem Federbusch. Niemand habe ihn je fressen gesehen, in Arabien sei er dem Sonnengott geheiligt, er werde 540 Jahre alt, um dann nach seinem Tode aus seinen Knochen wiederzuerstehen. ${ }^{17}$ Im „Physiologus “ wird der Vogel in Indien verortet. Bunt schillernd wie Smaragde, trage er eine Krone und sei unsterblich. Nach jeweils 500 Jahren fliege er in den Libanon, werde in Heliopolis brandgeopfert, um am nächsten Tag aus der Asche wieder aufzuerstehen. ${ }^{18}$

Es ist also hervorzuheben, dass der farbenfrohe Vogel in Margaretes Sammlung in eine Bewertungstradition integriert wurde, die perfekte Buntheit mit der Aura des Paradiesischen umgab. Der eigentümliche Vogel bestätigte wohl alle Aussagen der mythischen Beschreibungen: körperlos, kaum mehr ein richtiger Vogel, fast reine Feder und gleichsam die reine Farbe, fast nicht von dieser Welt - ohne Erdkontakt, weit entfernt am Nil, in Persien, in Indien oder in der Südsee, im Paradies angesiedelt: ein Wunderbares, fremder und schöner noch als der Papagei, eben der Vogel Gottes. Er war zwar ornithologisch klassifizierbar, insofern er den Vögeln überhaupt zugerechnet werden konnte, doch rückte er über die Topik des Unerreichbaren und Übernatürlichen aus der Alltagserfahrung ab: das machen nicht zuletzt die etwas späteren ornithologischen Werke deutlich, deren phantastischen Beschreibungskriterien für den Paradiesvogel sich deutlich von den viel konkreteren „normaler" Vögel unterscheiden. Dass selbst die ausgebalgten, betastbaren und gleichsam aus dem entfernten Bereich des Imaginären in die Wirklichkeit transferierten Exemplare überdies defigurierte Vögel waren, intensivierte diesen Aspekt des Wunderbaren wohl beträchtlich. Noch am Ende des 16. Jahrhunderts kommt diese Konno-

Sunt ibidem et aliae aves fuscae magnitudinis monedularum quae paradisi ideo vocantur, quia nescitur ubi nascantur, et unde nascantur, vel unde veniant, et illae mutant loca de tempore in tempus, sicut faciunt multa avium genera, de quibus in praehabitis mentionem fecimus sufficientem.

16 Vincentius [Bellovacensis]: Speculum quadruplex: sive speculum majus. I: Speculum naturale. Douais 1624 (Reprint Graz 1964), S. 1180. So auch übernommen in der Naturkunde des Thomas von Cantimpré (Helmut Boese (Hrsg.): Thomas Cantimpratensis: Liber de natura rerum. Editio princeps secundum codices manuscriptos, Tl. I : Text. Berlin u. New York 1973, V, 17, S. 185.)

17 Plinius, Hist. nat. X, 3-5.

18 Otto Schönberger (Hrsg.): Physiologus, Griechisch/Deutsch. Stuttgart 2001, Nr. 7 (dort auch weitere Nachweise zur antiken Überlieferung). 
tation des gleichsam Immateriellen zum Ausdruck, wenn im Emblembuch des Joachim Camerarius' der - als beständig in der Luft schwebender Federbausch gezeigte - Paradiesvogel zum Sinnbild geistiger Erhebung wird, welche sich irdischer Verstrickung entzieht. Der gemeinsame Kern der verschiedenen Interpretationsmuster liegt also darin, den Paradiesvogel in einer fremden Sphäre zu verorten, die gleichsam jenseitigen Charakter hat. Entscheidenden Anteil an dieser Einordnung des Paradiesvogels in das Reich des Wunderbaren hat seine Farbenpracht: Es mangele ihm an keiner Farbe, er inkarniert die Perfektion eines farbigen Vogels, schwerelos fliegend und von unglaublicher Farbintensität.

Lange zuvor schon, bereits seit der Antike, hatte eine ähnliche Wertschätzung den Grund dafür abgegeben, dass insbesondere Papageien zum begehrten Sammlungsobjekt vor allem von Fürsten geworden waren. ${ }^{19}$ Und es ist wohl auch eben der Buntheit zu verdanken, dass die Vögel schon früh zum Objekt gleichsam eigenwertiger Tierzeichnungen wurden. Bekannt ist in diesem $\mathrm{Zu}-$ sammenhang vor allem das sog. Skizzenbuch des Giovannino de'Grassi, des Mailänder Dombaumeisters am Ende des 14. Jahrhunderts. ${ }^{20}$ Entstanden zwischen ca. 1380 und ca. 1410, enthält es zahlreiche, offenbar teilweise auch nach der Natur gezeichnete Tierdarstellungen. Auffällig dabei ist, dass das Skizzenbuch die meisten Tiere zumeist monochrom in brauner Feder- und Pinselzeichnung festhält. Davon unterscheidet sich eine eigene, von der Werkstatt de'Grassis um 1400 ausgeführte Lage von drei Doppelblättern (fol. 9-14v), die hauptsächlich Vögel - darunter ein Wiedehopf und ein grüner Papagei - zeigt, die in den prächtigsten Farben detailliert widergegeben sind. Derartige eigenständige Bilder buntfarbiger Vögel konnten im 15. Jahrhundert zum geschätzten Sammlungsobjekt in fürstlichen Schätzen werden. So verzeichnet das Schatzinventar von René I. d'Anjou um die Mitte des 15. Jahrhunderts: Drei kleine Tüchlein, die in ein Zimmer gehängt werden sollen, von denen auf dem einen ein Pfau, ein Fasan, zwei Rebhühner, ein Affe und mehrere andere Dinge abgebildet sind. Auf dem anderen war ebenfalls ein Pfau gemalt, sowie ein Fasan, ein Wasservogel, zwei Nachtohreulen, usw. ${ }^{21}$ Und in unmittelbarer

19 Zuletzt und ausführlich zum Import von amerikanischen Papageien nach Europa: Pieper (Anm. 1), S. 245-271; Hermann Diener: Die „Camera Papagalli“ im Palast des Papstes. Papageien als Hausgenossen der Päpste, Könige und Fürsten des Mittelalters und der Renaissance, in: Archiv für Kulturgeschichte 49/1967, S. 43-97; Donald F. LACH: Asia in the Making of Europe. Mehrere Bde. Chicago u. London, vol. II: A Century of Wonder. Book one: The visual Arts (1970), S. 178-183.

20 Bergamo, Biblioteca Civica, Cassaf.1.21; Grulio Orazio Bravi / Maria Grazia ReCANATI (Hrsg.): Taccuino di disegni di Giovannino de Grassi. o. O. 1998.

21 In der Garderobe des Schlosses von Angers wurden verwahrt troys autres petites toilles à mectre en une chambre, dont en l'une a paint ung paon, ung feisant et deux perdriz, une chvèche [petit duc], ung cinge et pluseurs autres chouses; en l'autre est pareillement paint ung paon, ung fesant, ung oyseau de rivière, duex potz de grubelles et autres pluseurs chouses; en l'autre a escripz pluseurs petiz personnages à pié et à cheval, ung faulcon, ung connin blanc et 
räumlicher Nähe von Margaretes Paradiesvogel wurden 91 Blatt Papier mit Zeichnungen figurés de diverses bestes, oyseaux et autres painctures verwahrt. ${ }^{22}$ Das akribische Studium der reichen Farbvielfalt der Natur hat nicht zuletzt Albrecht Dürer zu seinen akkuraten Farbstudien von Blauracken und deren Flügeln sowie anderer Vögel veranlasst. ${ }^{23}$ Seine Vogelstudien widmen sich - soweit dies der Bestandüberlieferung $\mathrm{zu}$ entnehmen ist - keineswegs einem systematischen Erfassen von Vogelarten, sondern vor allem deren exotischen und farbenprächtigen Spezies. ${ }^{24}$

Angesichts der ohnehin bestehenden Wertschätzung von Papageien konnte man nur mit um so größerer Verwunderung konstatieren, dass es nun in der Neuen Welt von Papageien geradezu wimmelte - wie von Spatzen in Europa, heißt es bei Petrus Martyr d'Anghiera. ${ }^{25}$ Die Papageien fungieren gerade in den frühen Reiseberichten aus der Neuen Welt - von Columbus, Petrus Martyr d'Anghiera, Amerigo Vespucci usw. - als die wesentlichen Bestandteile, um den paradiesischen Charakter im Verein mit immergrünen Bäumen, blühenden Landschaften, höchster Fruchtbarkeit und beständigem Sonnenschein zu vermitteln. Auch hier war das Denkmuster älter: Schon in den literarischen Reisebeschreibungen nach Indien und China erhöht sich die Frequenz des Papageis bzw. des Phönix' auffällig, je weiter sich die Erzählung nach Osten bewegt. Im literarischen Stoff des mythischen Reichs des Priesterkönigs Johannes in Fernost etwa bilden die farbenprächtigen Vögel die Entsprechung zu den vielfarbig funkelnden Palästen und Gärten des sagenhaften Herrschers. ${ }^{26}$ Von den zahlreichen Varianten ist in unserem Zusammenhang vor allem das im Spätmittelalter weit verbreitete "Livre des merveilles" von Jean de Mandeville wichtig. ${ }^{27}$ Hier erscheinen die Papageien in ornithologisch genauer Beschreibung, die den älteren Naturkunden, etwa eines Thomas de Cantimpré bzw. eines Konrad von Megenberg, entsprechen. ${ }^{28}$ Die weitgehend realistische Schilderung des Vogels gewinnt ihre wunderhafte Komponente nicht aus sich selbst heraus, sondern aus

une ville et autre pluseurs choses. (A. LECOY DE LA MARCHE: Extraits des comptes et mémoriaux du Roi René ... Paris 1873, Nr. 642).

22 Michel.ant (Anm. 3), S. 96.

23 Koreny (Anm. 13), passim. Hervorzuheben ist vor allem Dürers Studie eines Blaurackenflügels (Wien, Graph. Sammlung Albertina, Inv. 4840, D 104).

24 Sebastian A. Killermann: Dürers Pflanzen- und Tierzeichnungen und ihre Bedeutung für die Naturgeschichte. Straßburg 1910, v.a. S. 71; Koneny (Anm. 13), S. 70-73 u. Nr. 22.

25 Hans Klingelhöfer (Hrsg.): Peter Martyr von Anghiera: Acht Dekaden über die Neue Welt. 2 Bde Darmstadt $1972-1973$, Bd. I, S. 31 u. 37.

26 Friedrich Zarncke: Der Priester Johannes. 2 Bde. Leipzig 1879 (Reprint Hildesheim New York 1980), I, S. 85 u. II, S. 145.

27 Christiane Deluz (Hsrg.): Jean de Mandeville: Le livre des merveilles du monde. Paris 2000 (=Sources d'histoire médiévale, 31), S. 437.

28 Robert Luff / Georg Steer (Hrsg.): Konrad von Megenberg: Das „Buch der Natur“, Bd. II. Kritischer Text nach den Handschriften. Tübingen 2003 
der Verortung des Vogels als Teil des prächtigen, von Edelsteinen funkelnden Palastes des Johannes. Um den schon lange bekannten indischen Papagei wird eine wunderbare Szenerie aus phantastischen Architekturen und einer Natur imaginiert, die aus den preziösesten Edelsteinen bestehen. Eben diese literarische Verortung des Papageis als einer in eine wunderhafte Umgebung eingefügten Realie dürfte sich auch bei der Betrachtung der Paradiesvogelbälge eingestellt haben. Um die konkrete Naturalie gruppieren sich die von den Reiseschreibungen alimentierten Bilder einer neuen Welt, die zwischen den Polen des Garten Edens und einer fremden exotischen Topographie mit wilden Tieren oszillieren.

Gilt also schon für den Papagei, dass er in seiner Farbigkeit einen überaus hohen Perfektionsgrad der Naturschöpfung darstellt, so ist dies noch mehr auf den Paradiesvogel anzuwenden. Hier liegt implizit die Vorstellung zugrunde, dass ein Kriterium der göttlichen Schöpfung auch ihre perfekte Farbigkeit gewesen sei. In Verbindung zu bringen ist dies beispielsweise mit dem bei Ez 28, 13 beschriebenen Paradies, das durch funkelnde farbige Steine geschmückt ist. Doch lässt sich gerade im Spätmittel auch die Denkfigur des malenden Schöpfergottes nachweisen, der das irdische Paradies mit kostbarsten Materialien, aufwendigsten Techniken und insbesondere in allen Farbtönen geschaffen hat. $\mathrm{Zu}$ verweisen ist etwa auf die neapolitanische Bibel des Alunno d'Alife aus dem Anfang des 14. Jahrhunderts, in der die Miniaturen Gottvater zeigen, welcher gleichsam als Buchilluminator das Bild der Schöpfung mit einem Pinsel malt. ${ }^{29}$ Für den burgundischen Bereich des ausgehenden 15. Jahrhunderts lässt sich die von dem Chronisten und Dichter Le Molinet verfasste Epitaphinschrift für den Maler Simon Marmion nennen. Die mimetischen Qualitäten des Malers werden hier verglichen mit der Exzellenz des peintre éternel et universel, also des Schöpfergottes. ${ }^{30}$ Eine ganz ähnliche Auffassung von Gott als oberstem artifice e pintor findet sich in der seit den zwanziger Jahren des 16. Jahrhunderts entstehenden ethnographischen Darstellung der Neuen Welt durch Fernández de Oviedo. ${ }^{31}$ Was den Paradiesvogel betrifft, liegen eben dieser Denkfigur schon die Definitionen der mittelalterlichen Enzyklopädisten zugrunde, die den Paradiesvogel als von Gott geschaffenen idealschönen Vogel kennzeichnen, dem keine Zierde fehle.

29 Christiane Kruse: Warum Menschen malen. Historische Begündungen eines Bildmediums. München 2003, S. 162-169.

30 Nö̈L Dupire (Hrsg.) : Les Faictz et dictz de Jean Molinet, 3 Bde. Paris 1936-39, Bd. 2, S. $824-25$.

31 J. Pérez de Tudela Bueso (Hrsg.): Conzales Fernández de Oviedo: Historia general y natural de las Indias, 1534-1557. Madrid 1959, Bd. IV, S. 330. S. a. Kart Kohut: Humanismus und Neue Welt im Werk von Gonzalo Fernándoz de Oviedo, in: Reinhard (Anm. 1), S. 65-88. 
Um so mehr musste auch das künstlerische Studium der Farbigkeit der Vögel im späten Mittelalter ein besonderer Prüfstein der Maler sein, die seit dem 15. Jahrhundert zunehmend das Ideal der perfekten und lebensvollen Imitatio bzw. des Übertreffens der Natur folgten. Wenn Dürer etwa in seinem Aquarell des Blaurackenflügels die Farbigkeit gegenüber dem natürlichen Aussehen des Vogels nochmals intensiviert, so ist darin ein derartiges Übertreffen der Natur im Medium der Farbigkeit zu sehen. Im Zusammenhang der Wahrnehmung der Neuen Welt lässt sich dies gleichsam als Topos nachweisen. Amerigo Vespucci hebt in seiner Beschreibung der Vogelwelt Südamerikas hervor, dass die Papageien in diesen Regionen in so grosser Vielfalt an Formen und Farben vorkommen, ,dass selbst ein vollendeter Meister der Malerei wie Polyklet [!] bei dem Versuch, diese zu malen, scheitern müsste ${ }^{32}$ Und im selben Zusammenhang stellt Vespucci Überlegungen an, ob das irdische Paradies eben nicht doch in diesen Regionen, also irgendwo in Brasilien, zu finden sei.

Gut vergleichbar damit erscheint der Papagei auch an prominenter Stelle in Dürers Adam-und-Eva-Stich aus dem Jahre $1504 .{ }^{33} \mathrm{Er}$ sitzt auf dem Zweig einer Bergesche - nach damaliger Auffassung der Lebensbaum -, den Adam mit seiner Rechten umgreift. Unmittelbar daneben hängt eine Schrifttafel, auf der Dürer seine Autorschaft in monumentaler Weise bestätigt (ALBERT ${ }^{9}$ DURER NORICVS FACIEBAT 1504) und damit kenntlich gemacht hat, dass er als alter deus den ideal schön proportionierten Menschen nachgebildet hat. Carsten-Peter Warncke hat darauf aufmerksam gemacht, dass der Papagei in eben diejenige Richtung blickt, in die auch die Schrifttafel gewendet ist und von wo das Licht erstrahlt, das die Szenerie beleuchtet. ${ }^{34}$ Der Papagei, Musterexemplar der Schöpfung, wird - so argumentiert das Bild - in seiner bildlichen Widergabe dem göttlichen Schöpfer gleichsam als Prüfstein perfekter künstlerischer Imitatio vorgeführt. Der idealen Proportioniertheit des ersten Menschenpaares entspricht die reine Farbigkeit des Papageis im Paradies. Hierbei spielt keine Rolle, dass der Kupferstich ja ein Schwarz-Weiß-Medium ist, denn er erhebt gerade bei Dürer den Anspruch, in seinem Modulationsreichtum mit den Möglichkeiten der Malerei zu konkurrieren. Bezeichnenderweise verfeinerte

32 Robert Wallisch (Hrsg.): Der Mundus Novus des Amerigo Vespucci (Text, Übersetzung und Kommentar) Wien 2002, S. 25. Den topischen Charakter der Aussage verdeutlicht der Umstand, dass Polyklet als angeblich größter antiker Maler, aber eben nicht als Bildhauer aufgeführt wurde.

33 Ratner Schoch U.A. (Hrsg.): Albrecht Dürer .Das druckgraphische Werk, Bd. I. Kupferstiche, Eisenradierungen und Kaltnadelblätter. München, London, New York 2001, Nr. 39; Anne-Marie Bonnet: Albrecht Dürer, „Adam und Eva“. Der Kupferstich von 1504, in: Kunsthistorische Arbeitsblätter 3, 2002, S. 51-60.

34 Carsten-Peter Warncke: Sprechende Bilder - sichtbare Worte. Das Bildverständnis in der frühen Neuzeit. Wiesbaden 1987 (=Wolfenbütteler Forschungen, Bd. 33), $305-313$. 
Dürer die ursprünglich kräftiger eingetragene Binnenzeichnung des Papageis im vierten, vorletzten Druckstadium. Es handelt sich gleichsam um die ins Medium des Kupferstichs übertragene prächtige Farberscheinung, wie sie Dürer in seinen Vogelaquarellen vor Augen führt. ${ }^{35}$

Exotischer und farbenfroher als der Papagei war der Paradiesvogel, zudem er aus noch entfernteren Gebieten stammte. Und wenn er auch kaum als tatsächlich aus dem Paradies kommend erachtet wurde, so trug er seinen Namen doch zurecht: Er stellte gleichsam einen Reflex des Paradiesischen, eine Möglichkeit idealer Naturschöpfung dar, die sich gerade in der perfekten Farbvariation manifestierte und mit den ersten Importen der Vögel auch konkretisierte. In der Rezeption des Paradiesvogels bzw. des Papageis überlagern sich also mittelalterliche und frühneuzeitliche Auffassungen: In der exotische Fremdheit und der an keine Materie gebundenen Farbenpracht galten die Vögel als ideale Musterexemplare der schönen Zierde der Schöpfung. Dies entspricht mittelalterlicher Auffassung. Doch in eben dieser Eigenschaft eigneten sie sich um 1500 nicht nur als exquisiter Sammlungsgegenstand im Zusammenhang der Entdeckung der Neuen Welt, sondern auch als ein Prüfstein einer Neubewertung der Malerei, die zunehmend ihre eigene Autonomie als Kunstgattung theoretisierte. Wenn das auch auf die göttliche Schöpfung anzuwenden war, so musste man das Künstlertum Gottes auch an der überseeischen Flora und Fauna - eben etwa dem Paradiesvogel - nachweisen können.

Lassen sich im Fall des Paradiesvogels nur indirekte Argumente für die vorgeschlagene Deutung bringen, so ist die Rolle des neuen Kunstdiskurses als universell anzuwendendes Instrument bei der Bewertung der Sammlungsstücke aus der Neuen Welt manifest. Die Kollektion Margaretes umfasste vor allem Kleidungsgegenstände aus Federn sowie Waffen und Kultgegenstände, insgesamt ca. 70 Stücke. Aus den genauen Beschreibungen in den Inventaren ist zu schließen, dass es sich um äußerst wertvolle Objekte handelte. Doch auffällig häufig tritt dabei der schiere Materialwert in Konkurrenz zu der Qualität der handwerklichen bzw. künstlerischen Anfertigung: Einer der Federmäntel wird etwa beschrieben als ung riche manteau beau et grant, garniz de IIIc LXVII besans d'ar, de larbeur d'ung sol de France pièce, bien ouvré, à ung bort de plume blanche, jointe d'ung bort noir. ${ }^{36}$ Eine Randbordüre erhält das nicht häufige Qualitätsurteil fort bien faicte. Besonders auffällig ist in diesem Zusammenhang, dass trotz der Verwendung der kostbaren Materialien die Stücke im Gegensatz zu

35 Campbell Dodgson: The Masters of Engraving: Albrecht Dürer. London u. Boston 1926, Nr. 39; Koreny (Anm. 13), passim. Vgl. auch Fritz Koreny (Hrsg.): Albrecht Dürer und die Tier- und Pflanzenstudien der Renaissance. Symposion 1985 (= Jahrbuch der Kunsthistorischen Sammlungen in Wien 82-83/1986-87).

36 Eichberger, Leben mit Kunst (Anm. 2), S. 180 ; Michelant (Anm. 3), S. 63. 
sonstigen Werken der Schatzkunst nur in einem Fall mit ihrem Gewicht verzeichnet sind. Es zählte offenbar nicht primär der Materialwert. ${ }^{37}$ In einigen Fällen ist überdies die fremde Provenienz mit der Qualität der handwerklichen Aufführung miteinander verbunden. Der Stil einer der Federmäntel wird deshalb als à la mode du pä̈s bezeichnet, ebenso zwei Kopfbedeckungen als en la manière $d u$ pays beschrieben. ${ }^{38}$ Qualitätsbewusstsein paart sich also mit einem vorgeblichen Wissen über fremde Bearbeitungstechniken. Selbst wenn wir nicht wissen, was über die genaue Provenienz der Werke bekannt war, muss man generell gerade bei Margarete ein prinzipielles Wissen über die Neue Welt wohl voraussetzen. Der Vorsitzende des spanischen Indienrates (Consejo de las Indias), Mercurio Arborio Gattinara, war zu Anfang der 20er Jahre Berater Margaretes für das Herzogtum Burgund gewesen. Außerdem war eben ihr einer der 1521 gedruckten Berichte über die Entdeckung der Neuen Welt gewidmet und befand sich in Margaretes Bibliothek. Es handelt sich um die vierte Dekade von "De nuper sub Domino Carolo reperits insulis ..." des spanischen Hofchronisten Petrus Marthyr d'Anghiera, auch er ein Mitglied im Indienrat. ${ }^{39}$

Es ist auffällig, wie sehr sich die in den Inventaren angewandten Kriterien mit denjenigen in den Reiseberichten und Briefen überschneiden. In der erwähnten Schrift des Petrus d'Anghiera etwa wird in der Beschreibung der Geschenke Moctezumas überdeutlich auf dem Primat des Prinzips des opus superat materiam abgehoben: ,Wenn in derartigen Kunstfertigkeiten talentierte Menschen je etwas Großes erreicht haben, werden jene Eingeborenen mit Recht den ersten Platz einnehmen. Ich bewundere weniger das Gold und die Edelsteine, sondern ich staune nur, mit welchem Fleiß und welcher Kunstfertigkeit das Material verarbeitet ist. Vielerlei Arten von Schmuckwerk habe ich bislang schon gesehen, .... Aber soweit ich urteilen kann, habe ich bisher nichts Schöneres erblickt, das in gleichem Maße dem Auge gefallen könnte. ${ }^{60}$ Auch an anderer Stelle ist die Hochschätzung von handwerklicher Perfektion omnipräsent: Bloßes Gold etwa bedeute den Einwohnern auf Kuba nichts, denn unverarbeitet erscheine es wie auch der rohe Marmor unansehnlich. Erst unter

37 Michelant (Anm.3), S. 64. Eingehend dazu Eichberger, Leben mit Kunst (Anm. 2), S. $179-185$.

38 Michelant (Anm. 3), S. 64 ; Eichberger, Leben mit Kunst (Anm. 2), S. 181.

39 Marguerite Debae: La bibliothèque de Marguerite d'Autriche. Essai de reconstitution d'après l'inventaire de 1523-1524. Louvain u. Paris 1995, Nr. 371 ; Michelant (Anm. 3), S. 92. Die daneben stehenden Bücher waren drei Stundenbücher, die französische Version der Teseida von Boccaccio, Chastelains Temple de Bocace und ein Bericht über den Einzug der Königin Claude de France in Paris, außerdem das Lobgedicht von Jean Lemaire de Belges auf Margarete, die Couronne margaritique. Im selben Raum befand sich auch das Kästchen mit dem Paradiesvogelbalg (Vgl. Michelant (Anm.3), S. 91-134; Debae (Anm. 39), 494-508.)

40 Kinngelhöfer (Anm. 25), Bd. I, S. 375, Eichberger, Leben mit Kunst (Anm. 2), S. 183. 
dem Meißel eines Phidias oder Praxiteles werde daraus ein schätzenswertes Werk. ${ }^{41}$ Ähnliches findet sich bei der Beschreibung des Palastes des Comogrus in den Vorbergen der Kordilleren. ${ }^{42}$ Dabei spielt auch die Angemessenheit zwischen Naturressourcen und hochstehender Bearbeitung eine entscheidende Rolle, ein Kriterium, das die Indigenen wie die Europäer anwenden würden: ,Wie jene Menschen an unseren Pfauen und Fasanen den Schweif bewundern würden, wenn sie ihn sähen, so entzückt uns das Gefieder ihrer Tiere, aus dem sie Federbüsche und Kopfputz herstellen und geschmackvoll verarbeiten. ${ }^{433}$ In eigenartiger Ambivalenz interpretiert Petrus Martyr die Bevölkerung der Neuen Welt zwar zum einen als kannibalische Wilde, zum anderen aber auch wiederholt als Exemplum begnadeter Menschen, die trotz mangelnder theoretischer Bildung und metallener Werkzeuge kraft ihres Ingeniums höchst qualitätvolle Artefakte zu schaffen imstande sind. So beschreibt er wiederholt die Behausungen der Indianer gemäß den bei Vitruv überlieferten Merkmalen der Urhütten primitiver Menschengruppen: In die Erde gerammte Stämme mit Dachwerk aus Zweigen und Blättern. ${ }^{44}$ Wesentlich daran ist, dass dieser Initiumstopos bei Vitruv verbunden ist mit der erstmaligen Anwendung von Sprache und Intelligenz unter den Menschen. Der sich stetig verbessernde Hausbau bildet insofern das Paradigma einer sich beständig perfektionierenden Gemeinschaft. ${ }^{45}$ Deren Gipfelpunkt in der Neuen Welt wird auch ausführlich und mit Ehrfurcht in der Schilderung der Stadt Tenochtitlan mit dem Palast Moctezumas beschrieben. In Weiterführung von Elementen der der italienischen Idealstadtvorstellungen des 15. Jahrhunderts - von Leon Battista Alberti, Filarete und Francesco di Giorgio Martini - wird die prosperierende Hauptstadt mit ihrer zentralen und geraden Hauptstraße genau geschildert. Vollendente Handwerker schaffen hier, trotz des Fehlens von Eisen und Stahl, Vorzügliches; ein reges kaufmännisches und kultisches Leben herrscht, auch dank eines freigiebigen Fürsten; die Paläste sind größer als alle vergleichbaren spanischen Anlagen, enthalten allesamt Höfe und Gärten und glänzen von Säulen aus Jaspis und weißem Marmor; hohe Arkaden tragen Sonnenterrassen; in seinen Landhäusern hält Moctezuma in den einen Bucklige, Einarmige, Zweiköpfige und Albinos, in anderen Tiere und Vögel usw. Die hellglänzende ideale Pracht wird indessen durch eine abscheuliche Sünde verunreinigt: die allgegenwärtigen Menschenopfer, deren Blutströme stinkend auf dem Alabaster kleben, wie Petrus Martyr oftmals drastisch kontrastiert - ohne die Bauten selbst je gesehen zu

41 KLINGELhöFer (Anm. 25), Bd. I, S. 51.

42 Kingelhöfer (Anm. 25), Bd. I, S. 159-163.

43 Kingelhöfer (Anm. 25), Bd. I, S. 375.

44 Klingelhöfer (Anm. 25), Bd. I, S. 35-36 und passim.

45 Curt Fensterbusch: Vitruv, zehn Bücher über Architektur, Darmstadt 1964 ( lat.-dt. ), Buch I, 1. 
haben! $!^{46}$ Man hat darauf verwiesen, dass auch die bildliche Umsetzung des Stadtplanes von Tenochtitlan, die 1524 als Holzschnitt im Druck der Briefberichte von Hernan Cortés erschien, offenbar derartig vorbildlich erschien, dass sie das Vorbild für den quadratischen Idealstadtplan abgab, welchen Dürer in seinem Festungsbautraktat vorstellte. ${ }^{47}$

Recht ähnliche Argumente finden sich auch bei Cortés, wenn dieser in einem seiner Briefe an Karl V. schreibt: ,Die Gold- und Silberarbeiten sind so naturgetreu, dass es keinen Gold- oder Silberschmied in der ganzen Welt gibt, der es besser machen könnte. Was die Steine anbetrifft, so kann man sich kein Instrument vorstellen, womit man sie so vollkommen hätte ausführen können. Und die Federarbeiten: man kann die Natur weder in Wachs, noch mit Stickereiarbeiten so vollkommen imitieren. ${ }^{\text {‘48 }}$ Eine nämliche positive Wertschätzung ist auch den Chroniken von Gasparo Contarini, Francisco Lopez de Gómara und Bernal Díaz del Castillo zu entnehmen: Beim letzteren sind es insbesondere die wohlproportionierten riesenhaften Tempelanlagen und die begabten Maler, die die Bewunderung des Chronisten hervorrufen: Einige der Maler seien Apelles, Michelangelo oder Berruguete ebenbürtig. ${ }^{49}$

Technische Perfektion und ,imitatio naturae' sind hier die Grundforderung einer nicht nur handwerklich regelgerechten, sondern darüber hinausgehenden künstlerischen Gestaltung der Objekte. Im Gebiet von Architektur und Städtebau erweisen sich analog die Kriterien von technischer Meisterschaft, Hygiene, Regelhaftigkeit und Angemessenheit als Grundlage der Bewunderung. Diese wird indessen nicht umgesetzt in ein ethnographisches Argument, um die $\mathrm{Zi}$ vilisiertheit der Indianer argumentativ herauszuarbeiten. Kannibalismus, Unzucht und Menschenopfer kontrastieren unvermittelt mit dem hohen Standard der kunsthandwerklichen Produktion. ${ }^{50}$ Auch diese Ambivalenz scheint der Topik der älteren mythischen Beschreibungen „Indiens" verpflichtet zu sein, wo das sagenhafte ideale Reich des Priesterkönigs Johannes ebenfalls mit wilden

46 KLINGELHÖFER (Anm. 25), Bd. II, S. 4449-53.

47 Albrecht Dürer: Etliche underricht, zu befestigung der Stett, Schloß, und flecken, Nürnberg 1527; ERwin WaLter PaLM: Tenochtitlán y la ciudad ideal de Dürer, Journal de la Société des Américanistes, n.s., 40/1951, 59-66. Hanno-Walter Kruft: Geschichte der Architekturtheorie. München $1991^{3}$, S. 123-124

48 Mario Hernández (Hrsg.): Hernán Cortés, Cartas de relación. Madrid 1988 (= Cronicas de America, Bd. 10), hier zit. n. Erchberger, Leben mit Kunst (Anm. 2), S. 183.

49 Miguel Léon-Portilla (Hrsg.): Bernal Díaz del Castillo: Historia verdadera de la conquista de la Nueva España, 2 Bde. Madrid 1984 (= Cronicas de America 2a), Bd. I, S. 328 u. 332-334; José LuIS de RojAs (Hrsg.): Francisco Lopez de Gómara: La conquista de México. Madrid 1987 (= Cronicas de America, 36), S. 86, 188-190. Allg. dazu: Keen (Anm. 1), S. 56-70; Honour (Anm. 1).

50 Hans-Joachim König: Verständnislosigkeit und Verstehen, Sicherheit und Zweifel: Das Indiobild spanischer Chronisten im 16. Jahrhundert, in: Bitterli/Schmitt (Anm. 1), $37-62$. 
Männern und Kannibalen bevölkert ist. Außerdem ist hervorzuheben, dass einige Chronisten - so etwa Petrus Martyr - die Stätten und Produkte der Neuen Welt nicht aus eigener Anschauung kannten. Wenn diese doch mit den dezidierten positiven Werturteilen beschrieben werden, so zeigt sich hier eine neue, offenbar recht weit verbreitete Geschmackskultur, die begründende Argumente als ekphratische Topoi anzuwenden hat.

Vor diesem Hintergrund heben sich diejenigen Begründungsmuster $\mathrm{ab}$, die die Wertschätzung der indianischen Kultur in der Tat in zivilisationstheoretische Argumente einbeziehen. Gute Kunst bzw. die prinzipielle Fähigkeit, sie zu schaffen, kann als Erzeugnis bzw. Ausdruck idealer menschlicher Gemeinschaft gewertet werden. Eben dieser Aspekt wird prägnant von Bartholomé de Las Casas in seinen harschen Kritiken an dem Wüten der Spanier in der Neuen Welt betont; verwiesen sei etwa auf die 1550 verfasste, aber lange zuvor vorbereitete „Apologetica Historia Sumaria“. Wie die Europäer letztlich von Adam abstammend, seien auch den Indianern wesentliche anthropologische Fähigkeiten eigen; etwa seien sie soziale Wesen mit politischer Klugheit und familiärer Moral; sie verfügten über die Möglichkeit zu Enthaltsamkeit und Mäßigung sowie eine natürliche Geschicklichkeit als Jäger und in der Anfertigung von Kleidung. Weiterhin handele es sich um körperlich gut gebaute Menschen mit der richtigen Mischung der Körpersäfte; und ihnen sei die ,Güte inneren Vermögens und ihrer Organe, nämlich des Gemeinsinns, der Vorstellungskraft, der Phantasie und des sensitiven Gedächtnisses' gegeben. ${ }^{51}$

Die hier genannten und uns vor allem interessierenden Fähigkeiten von Vorstellungskraft und Phantasie als anthropologische Grundkonstanten der Schaffung und Beurteilung von Kunst aus Übersee ist nun ansatzweise bereits für die Zeit um 1520 in Bezug auf die Stücke aus Übersee belegt. Das beste und viel zitierte Beispiel in diesem Zusammenhang bietet wiederum Albrecht Dürer, der auf seiner Reise in die Niederlande auch den in Brüssel ausgestellten Moctezuma-Schatz besichtigt. Der Bewunderung voll vertraut er seinem Tagebuch folgendes an: auch hab ich gesehen die ding, die man dem König aus dem neuen gulden land hat gebracht: ein ganz guldene sonne, einen ganzen klafter breit, desgleichen zwo kammern voll derselbigen rüstung, dergleichen von allerlei ihrer waffe, harnisch, geschütz, wunderbarlich wahr, selzsamer Kleidung, bettgewand und allerlei unserbarlicher Ding zu manniglichem gebrauch, das so viel schöner an $z u$ sehen ist dan wunderding. Diese ding sind alle köstlich gewesen, das man sie beschäzt umb hundert tausend gulden werth. Und ich hab aber all mein lebtag

51 Mariano Delgado (Hrsg.): Bartolomé de Las Casas: Werkauswahl, Bd. 1: Missionstheologische Schriften. Paderborn, München, Wien, Zürich. 1994, hierin: „De unico vocationis modo omnium gentium ad veram religionem“, S. 97-335, hier S. 104-105. S. a. ibid. Bd. 2: Historische und ethnographische Schriften, hierin: „Apologetica Historia Sumaria“, S. 325-512, hier S. 329 u. 405-408. S. a. KeEn (Anm. 1), S. 92-104. 
nichts gesehen, das mein herz als erfreut hat als diese ding. Dann ich hab darin gesehen wunderliche künstliche ding und hab mich verwundert der subtilen ingenia der menschen in fremden landen. Und der ding weiß ich nit auszusprechen, die ich do gehabt hab. ${ }^{52}$

Christian Feest hat den topischen Charakter der Werturteile Dürers deutlich gemacht, der nicht dazu verleiten sollte, hier die Wahrnehmung eines spezifischen Kunstcharakters der Werke zu suchen. ${ }^{53}$ Gleichwohl ist interessant, dass innerhalb der Topik die wundersame Qualität der Kunstgegenstände mit der subtilen, ingenia' der Meister verbunden wird. Der Begriff, am ehesten mit Talent/Können zu übersetzen, ist gemäß der Texte Dürers das Pendant zu ,Kunst', also der regelgerechten Beherrschung eines Metiers. Beides reguliert den reichen Vorrat an imaginären Vorstellungsbildern, die im Künstler entstehen. ${ }^{54}$ Hier ist implizit abgehoben auf eine während des 15. Jahrhunderts - spätestens mit dem Malereitraktat von Cennino Cennini - immer mehr auf die Bedeutung der bildlichen Imaginationskraft abhebenden Kunsttheorie. Talent und Können stellen Grundvoraussetzung dafür dar, die bildergenerierende Imagination bzw. Phantasie in materielle, künstlerisch qualitätvolle Produkte umzusetzen. ${ }^{55}$ Dabei obliegt es nach mittelalterlicher Vorstellung der rationalen Kraft der ,vis cognitiva' und der ,vis estimativa', kohärente Bilderscheinungen zu produzieren, und diese nicht in die unkontrollierbaren Wahnvorstellungen der ,phantasmata abzugleiten zu lassen. ${ }^{56}$ In diesen Kategorien wird also auch die künstlerische Produktion aus der Neuen Welt eingeordnet, der neue Diskurs über Kunst erprobt gleichsam seine universelle Anwendbarkeit.

52 Hans Rupprich: Albrecht Dürer: Schriftlicher Nachlaß. 3 Bde. Berlin 1956-1969, Bd. I, S. 155.

53 FEest, „Selzam ding ..." (Anm. 1).

54 Erwin Panofsky: Dürers Kunsttheorie, vornehmlich in ihrem Verhältnis zur Kunsttheorie der Italiener. Berlin 1915, S. 166-180.

55 Panofsky (Anm. 54); allg. dazu Ulrich Pfisterer: Die Kunstliteratur der italienischen Renaissance. Eine Geschichte in Quellen. Stuttgart 2002, passim.

56 Albertus Magnus : De Anima, v. a. Lib. III, tract. 1, cap. 3, cap. 6-9. Vgl. dazu Marie Dominique Chenu O. P.: Imaginatio. Note de lexicographie philosophique médiévale, in: Miscellanea Giovanni Mercati (Studi e testi, 122), Bd. II, Vatikanstadt 1946, S. 593-602; Murray Wright Bundy: The Theory of Imagination in Classical and Mediaevel Thought, Urbana 1927 (=Univ. of Illinois Studies in Language and Litterature 12/1927, n ${ }^{\circ} 2-3$ ); MARTIN KEMP: From Mimesis zu Fantasia: the Quattrocento Vocabulary of Creation, Inspiration and Genius in the Visual Arts, in: Viator 9/1977, S. 347-98; Douglas Kelly: Medieval Imagination. Rhetoric and the Poetry of Courtly Love. Madison u. London 1978, v. a. S. 26-56; Christian Freigang: „Fantaisie et Ymaginacion": Selbstreflexion von Höfischkeit am provençalischen Hof unter René I., in: Christian Freigang/Jean-Claude Schmitt (Hrsg): Hofkultur in Frankreich und Europa im Spätmittelalter, Berlin 2005 (= Passagen/Passages, Bd. 11), S. 209-243. 
Gleichsam als Beleg dazu kann Dürers Verbildlichung eines Ureinwohners aus Amerika oder „Indien“ notiert werden. Zu sehen ist ein solcher auf der Seite zu Psalm 24 im Gebetbuch für Kaiser Maximilian in der Bayrischen Staatsbibliothek in München, dessen Bordürenschmuck 1515 von Dürer - und ihm folgend Altdorfer, Cranach, Breu u. a. - ausgeführt wurde. ${ }^{57}$ Hier erscheint einer der wohl proportionierten Bewohner einer - nicht präzise im ethnographischen Sinne zu konkretisierenden - fremden Welt, angetan mit einem Federschmuck auf dem Kopf und um den Hals sowie einem Federrock um die Hüften. ${ }^{58}$ Die Kampfkeule in seiner Rechten ist mit realen Waffen $\mathrm{zu}$ identifizieren. Die ponderierte Körperhaltung und die fein proportionierten Körperformen lassen erkennen, dass es sich um mehr als einen edlen Wilden, eher um einen recht nahen Abkömmling des ersten, ideal schönen Menschenpaares handelt. Und mehr noch, die kleinteilig ausgeführte Federgewandung, der dekorierte Schild usw. legen Zeugnis von dem hohen handwerklichen Niveau der hier angedeuteten Zivilisation ab. Mit der Vorführung der fremden Welt geht der Randschmuck auf den hier einsetzenden Psalm 24.1 ein: Domini est terra et plenitudo eius orbis terrarum et universi qui habitant in eo: ,Dem Herrn gehört die Erde und was sie erfüllt, der Erdkreis und seine Bewohner. Gott als Herrscher und letztlich Schöpfer einer idealschönen Erde anzudeuten, mag die positiven Aspekte der hier vorgeführten Fremde erklären. - Die den Psalmvers abschließende bzw. den folgenden Psalm 45 kommentierende Illustration stellt als Pendant zum „Indianer“ einen Türken mit Kamel dar. Dies ergänzt also den Aspekt der christlichen Weltbeherrschung und rekurriert auch auf eine politische Programmatik Maximilians. ${ }^{59}$

Die Randillustrationen sind indessen insgesamt von einer erstaunlichen Heterogenität und lassen sich deshalb nicht nach einem einheitlichen Verweissystem auf die zugehörigen Gebetstexte beziehen. Wie jüngst Magdalena Bushart eruiert hat, spielen mnemotechnische Aspekte dabei eine wichtige Rolle. Einzelne Textbestandteile werden in verschiedenster Weise bildlich markiert und kommentiert: Dies kann im Sinne von Ähnlichkeiten oder Kontrastierungen zwischen Text und Bild oder auch über eine bildliche, von Details ausgehende Assoziation geschehen. ${ }^{60}$ Dabei bleibt indes, wie Friedrich

57 München, BSB, $2^{\circ}$ L. impr. membr. 64, f ${ }^{\circ}$ 41. Hinrich Sievering (Hrsg.): Das Gebetbuch Kaiser Maximilians. Der Münchner Teil mit den Randzeichnungen von Albrecht Dürer und Lucas Cranach d. Ae. Rekonstruierte Wiedergabe. München 1987; Sieveking plädiert mit Hinweis auf eine vergleichbare Darstellung von Eingeborenen aus Sumatra in einer Zeichnung Jörg Breus dafür, den Krieger nicht wie bisher als Indianer sondern als Malaisier zu deuten (S. XXIII-XXIV).

58 FEEST, „Selzam ding ..." (Anm. 1). S. 116-117.

$59 \mathrm{~F}^{\circ}$ 42v. SieveKing (Anm. 57), S. XXIV.

60 Magdalena Bushart: Sehen und Erkennen. Albrecht Altdorfers religiöse Bilder. München u. Berlin 2004, S. 159-192; Ewald M. Vetter/Christoph Brockhaus: 
Teja Bach analysiert hat, die mediale Qualität dieser Bildkommentare gezielt heterogen und oszillierend. ${ }^{61}$ Die figürlichen Darstellungen sind von eigentümlichen Ranken und Arabeskenlinien umgeben, die teilweise aus den szenischen Abbildungen hervorgehen und sich in unidentifizierbare Gegenstände und Vexierbilder verwandeln. Mitunter nimmt die Ranke Konturlinien der figürlichen Darstellungen oder auch Schwünge der kalligraphischen Buchstaben des Textes auf. Der Indianer und die anderen Hauptbilder der Marginalillustrationen sind somit eingebunden in wuchernde, sich in ihrer Abbildqualität beständig verändernde Ranken und Linien, die entwicklungsgeschichtlich aus den älteren Randillustrationen aus vegetabilen Ornamenten, Drolerien, burlesken Szenerien und kuriosen Fabelwesen abzuleiten sind. In zahllosen Beispielen übernehmen die Bildchen hierbei die Rolle einer respektlosen oder burlesken Kommentierung von Motiven und inhaltlichen Sequenzen aus dem Textspiegel oder dessen Initiale. Um die Kontrastfunktion der meist ungeordneten, inkohärenten, oft zügellosen Welt deutlich zu machen, stellen die Maler häufig gerade Monster, Fabelwesen und groteske Szenen dar. ${ }^{62}$ Man kann dieses Verfahren auch so deuten, dass bei deren bildlicher Erfindung eine Phantasie am Werke ist, der die ordnende Kraft der, vis cognitiva' abhanden zu kommen und in die sinnesverwirrenden gefährlichen Phantasmata abzugleiten scheint - im Gegensatz zum geordneten, orthodoxen Text bzw. der Hauptillustration in der Seitenmitte. Erst in dieser bildgenerierenden Aktivität der Vorstellungskraft entsteht die gezielt originelle, Aufmerksamkeit heischende Kommentierung des Textes durch das Bild.

Besonders deutlich wird dies in den unmittelbaren Vergleichstücken zu Dürers Randillustrationen, insbesondere einer Zeremonialhandschrift für Philipp von Kleve ${ }^{63}$ und dem in den 70er Jahren des 15 . Jahrhunderts entstandenen sog. "Stundenbuch der Maria von Burgund“. ${ }^{64}$ Hier sind es Jagd-

Das Verhältnis von Text und Bild in Dürers Randzeichnungen zum Gebetbuch Kaiser Maximilians, in: Anzeiger des Germanischen Nationalmuseums 1971/72, S. $70-121$.

61 Friedrich Teja Bach: Struktur und Erscheinung. Untersuchungen zu Dürers graphischer Kunst. Berlin 1996.

62 Michael Camille: Image on the Edge. The Margins of Medieval Art. London 1992.

63 Wien, Österreichische Nationalbibliothek, Cod. S. n. 2616 (Отто Pächt, DagmarTross: Flämische Schule II, Textband. Wien 1990 (= Die Illuminerten Handschriften und Inkunabeln der Österreichischen Nationalbibliothek, Bd. 7), S. 36-38.

64 Wien, Österreichische Nationalbibliothek, Cod. 1857; Franz UnTERkircher (Hrsg.): Das Stundenbuch der Maria von Burgund. Codex Vindobonensis 1857 der Österreichischen Nationalbibliothek. Graz 1993; Pächt/Thoss (Anm. 63), S. 69-85. Zu den Vorbildern Dürers vgl. a. Hans Christoph von TAvel: Die Randzeichnungen Albrecht Dürers zum Gebetbuch Kaiser Maximilians, in: Münchener Jahrbuch für Bildende Kunst 16/1965, S. 55-120; Bodo Brinkmann: „Marginalia“ on Dürer. Netherlandish sources for his illustrations in the Prayerbook of Emperor Maximilian, in: Meddeleeuwse handschriftenkunde in de Nederlanden 1988, Grave 1989 (Nijmeegse codicologische Cahiers, Nr. 10-12), S. 183-197. 
szenen, spinnende Mädchen, Tiere, Fabelwesen usw., die den Text begleiten. Die Bezüge zu Dürer bestehen zum einen darin, dass sich viele der Marginalillustrationen als assoziative Verwandlungen von Motiven aus den zugehörigen Hauptbildern verstehen lassen. Im Kalenderteil etwa erscheint das Instrument des Sternbildes Waage auch als Spielzeug eines Affen ( $f^{\circ} 10 / 10 v$ ), oder das Motiv eines Fisches aus dem entsprechenden Sternzeichen wird zur Beute eines bunten Vogels, der seinerseits von einem aus dem Papiergrund auftauchenden Schlund bedroht wird ( $f^{\circ} 3 \mathrm{v}$ ). Immer wieder bilden spielende Affen oder höfische Szenen einen Kontrast zu den Gebeten im Text. Die Darstellung von Unsinn und frivolem Vergnügen konstrastiert zu der aufmerksamen Devotion, die die Texte erheischen. Auffälligerweise spielen hier - wie generell in der burgundischen Buchmalerei dieser Zeit - auch zahllose bunt schillernde Vögel eine bestimmende Rolle: Pfaue, Fasane und nicht identifizierbare Vögel, gleichsam ins Irreale verzogene Variationen der oben genannten prächtigen Vogelbilder, die das höfische Ambiente markieren. Hier begegnen sich also gleichsam Paradies und Alltag, Hölle und Spiel, offensichtlich Produkte der assoziierenden Phantasie. - Zum anderen macht der Künstler des burgundischen Stundenbuchs ähnlich wie Dürer die schweifende Bildschöpfung zum Bildthema: Aus Buchstabenhasten werden Ranken, um sich zu verwandeln in Ornamentlinien- und Schwünge, auf denen Figuren reiten, sich umschlingen lassen usw. Immer wieder entstehen in der feinst und leicht geführten Linie wie zufällig Gesichtsprofile, die in einigen Fällen respektlos Luftblasen auszustoßen scheinen. Der Kalligraph wird zum Ornamentzeicher, zum Portraitisten und zum Maler und reiht dies scheinbar zufällig in die sichere Führung seiner Hand auf. ${ }^{65}$ Imagination und Phantasie, der bildproduktiven Kraft ist hier scheinbar freier Lauf gelassen, ein Lauf, der gefährlich nahe an das Phantasmata reicht, würde er nicht immer durch Hauptbild und -text gezügelt bzw. auf ein Zentrum geordnet werden.

Interessanterweise sind die beiden genannten Handschriften mit Maximilian I. in Verbindung zu bringen: Das Zeremoniale Philippes von Kleve wurde 1481 unter der Leitung des Wappenherolds Maximilians redigiert, und von dem Stundenbuch ist wahrscheinlich zu machen, dass es sich am Hof des Kaisers befand. Es sei indessen dahingestellt, ob sich Dürer direkt von diesen Illustrationen inspirieren ließ. Sicherlich ist aber in dem metamorphotischen Oszillieren der Zeichnungsmedien eine wichtige Gemeinsamkeit zu entdecken. Dürer hat aber darüber hinaus die schweifende Phantasie, welche sich der Lustbarkeit und der Angst hingibt und in der ungezügelten Linie Ausdruck gewinnt, auch in seiner,Engelsmesse von 1500 oder um 1515 in eine ironische,

65 B ACH (Anm. 61), S. 256-273. 
aber programmatische Zeichnung umgesetzt. ${ }^{66}$ Hier sieht man - prinzipiell vergleichbar der Bilderwelt auf einer Seite eines illuminierten Gebetbuches - ein buntes, offensichtlich ungeordnetes Treiben von Fabelwesen und Dämonen zwischen Engeln und religiösen Szenen, welches sich während einer Messe zuträgt. Dargestellt ist die Interferenz von frommen Gedanken und „unkeuschen“ Wahnbildern, wie sie beim unaufmerksamen Teilnehmer der Messe entstehen. Auf der Schrifttafel im Vordergrund ist handschriftlich in Dürers Kursive notiert Do schreibt hrein was ihr wollt, darunter folgen Linienarabesken, halb Schrift, halb ornamentale Ranken. Sie sind, analog zu der inkohärenten Bilderwelt darüber, bildgewordene Produkte der ungezügelten, beliebig zeichnenden Phantasie, die nicht ein Thema kohärent behandelt und entsprechend die Zeichnung ohne eindeutigen Titel lassen muss. Ähnliche negativ konnotierte zeitgenössische Verbildlichung des freien Laufs der Phantasie sind zu benennen, etwa eine Darstellung des guten und des schlechten Beters in einem Holzschnitt des 15. Jahrhunderts, wo dem unkonzentrierten Beter eine Vielzahl lasziver Bilder zugeordnet ist, freilich ohne dass dies in der schweifenden Linie bildnerisch reflektiert ist. ${ }^{67}$ In den beiden Gebetbüchern und dem Zeremoniale indessen ist die schweifende Phantasie in der zügellosen und sich verwandelnden Linie als Produktionsprozess selbst Bild geworden.

Worin liegt hierbei der Bezug zur Rezeption der Neuen Welt? Zum einen bestätigen die Randillustrationen die Selbstthematisierung der Phantasie als bildgenerativer Kraft, die im Geist des ,artifex' entsteht. Nicht die regelgerechte Ausführung eines handwerklichen Problems oder die Einhaltung eines Formenund Musterkanons geben hier die Grundlage der Beurteilung ab. Und entsprechend werden auch das fremde Federwerk und die Kultgegenstände der Neuen Welt in derartiger Weise positiv beurteilt; nämlich als Produkte, bei denen ,ingenium' und , ars' aufgewandt worden waren, um Imagination umzusetzen. Zum anderen aber fällt - einen Gedanken Michael Camilles weiterführend - auf, dass in gewisser Weise die Marginalillustrationen thematisch mit dem Bild der Neuen Welt übereinstimmen. ${ }^{68}$ Die Ränder der Vorstellungswelt, die Phantasmata, überlagern sich mit den Rändern der irdischen Welt: Paradiesvögel, Fabelwesen und wuchernde Pflanzen, nackte Menschen, Wilde, schließlich eine naive oder krude Ordnung: all das sind Motivübereinstimmungen und Strukturmerkmale, die sich in den Bildern am Rand der Buchseite und den Vorstellungsbildern von der Peripherie der Erdtopographie eigen-

66 Rennes, Musée des Beaux Arts; Friedrich Winkler: Die Zeichnungen Albrecht Dürers, Bd. I, Berlin 1936, Nr. 181.

67 В АCH (Anm. 61), S. 224-227.

68 Camille (Anm. 62). 
tümlich überdecken. ${ }^{69}$ Das Wirken der göttlichen Imagination an den Rändern der Welt - wo farbenprächtige Vögel neben den Missgeburten der Natur leben entspricht insofern der „freien Linie als Keimbahn der Bildsynthese der Vorstellungskraft" (Fr. T. Bach) in Dürers Gebetbuchillustrationen. Der Paradiesvogel und die schweifende Phantasie stellen somit Optionen dar, mit denen weniger das Fremde als vielmehr das Eigene imaginär erkundet wird. Hier bestätigte sich vielfältig ein neu entstandenes Verständnis der künstlerischer Produktion und vermochte insofern, die Appropriation der fremden Kulturen voranzutreiben.

Abbildungsnachweise: 1: Erlangen, Graph. Sammlung der Universität, Inv. B 164 II.C.4; nach Fritz Koreny: Albrecht Dürer und die Tier- und Pflanzenstudien der Renaissance. München 1985. - 2: Wien, Graph. Sammlung Albertina, Inv. 4840 (D 104), nach Koreny (wie 1). - 3: nach Reiner Schoch, Matthitas Mende, Anna Scherbaum: Albrecht Dürer. Das druckgraphische Werk, I. München, London, New York 2001. - 4: nach Hinrich Sievering (Hrsg.): Das Gebetbuch Kaiser Maximilians. Der Münchner Teil mit den Randzeichnungen von Albrecht Dürer und Lucas Cranach d. Ae. Rekonstruierte Wiedergabe. München 1987. - 5: nach Franz UnTERKircher (Hrsg.): Das Stundenbuch der Maria von Burgund. Codex Vindobonensis 1857 der Österreichischen Nationalbibliothek. Graz 1993. - 6: Rennes, Musée des Beaux Arts; nach Friedrich Winkler: Die Zeichnungen Albrecht Dürers, Bd. I, Berlin 1936.

69 Las Casas beschreibt konkret körperliche „Missbildungen“ einer Ethnie als Phantasmata der Natur (Delgado (Anm. 51), S. 378-379). 


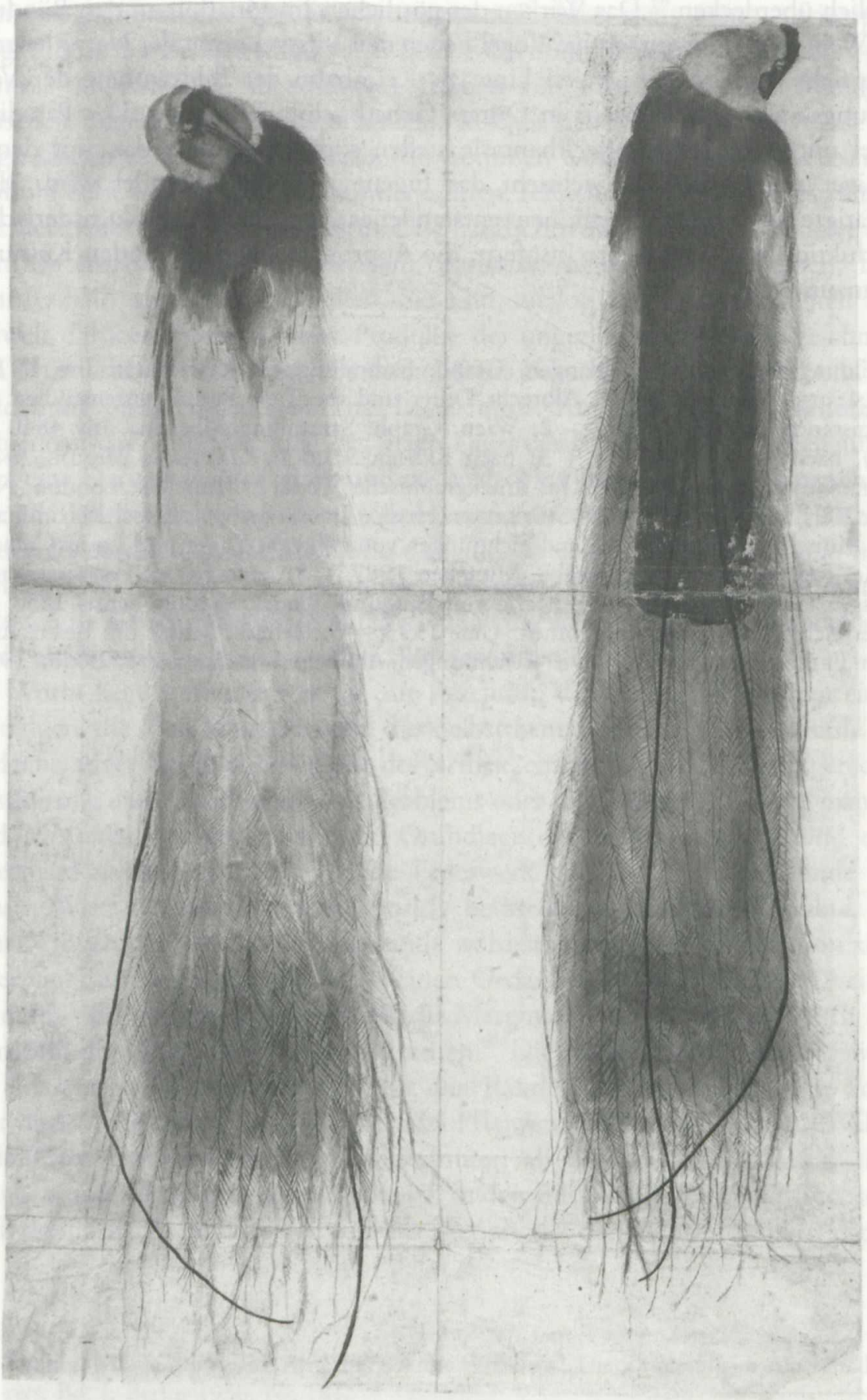

Abb. 1 Paradiesvögelbälge, Aquarell, 2. Hälfte d. 16. Jh. 


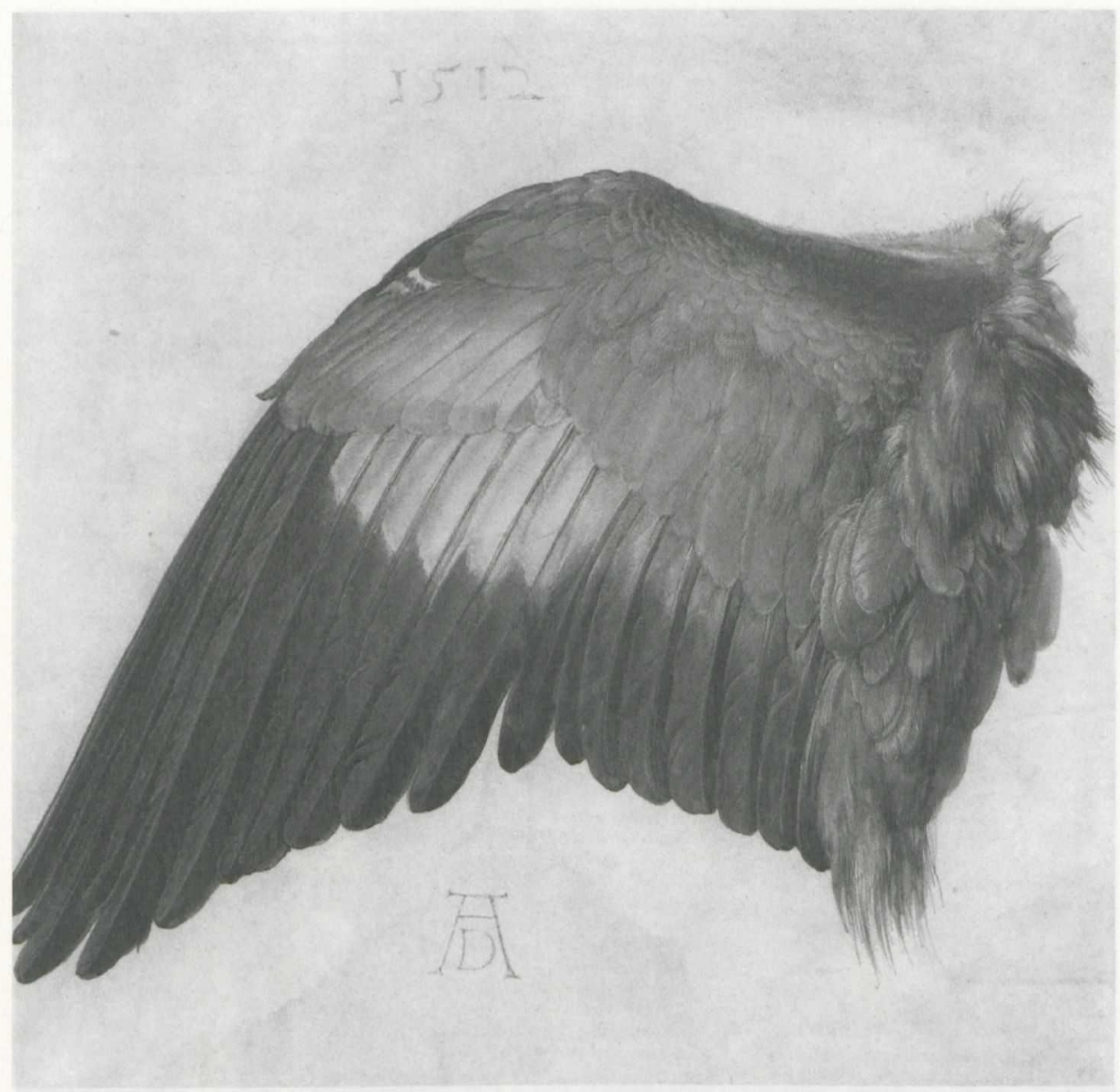

Abb. 2 Albrecht Dürer, Blaurackenflügel, Aquarell und Federzeichnung 


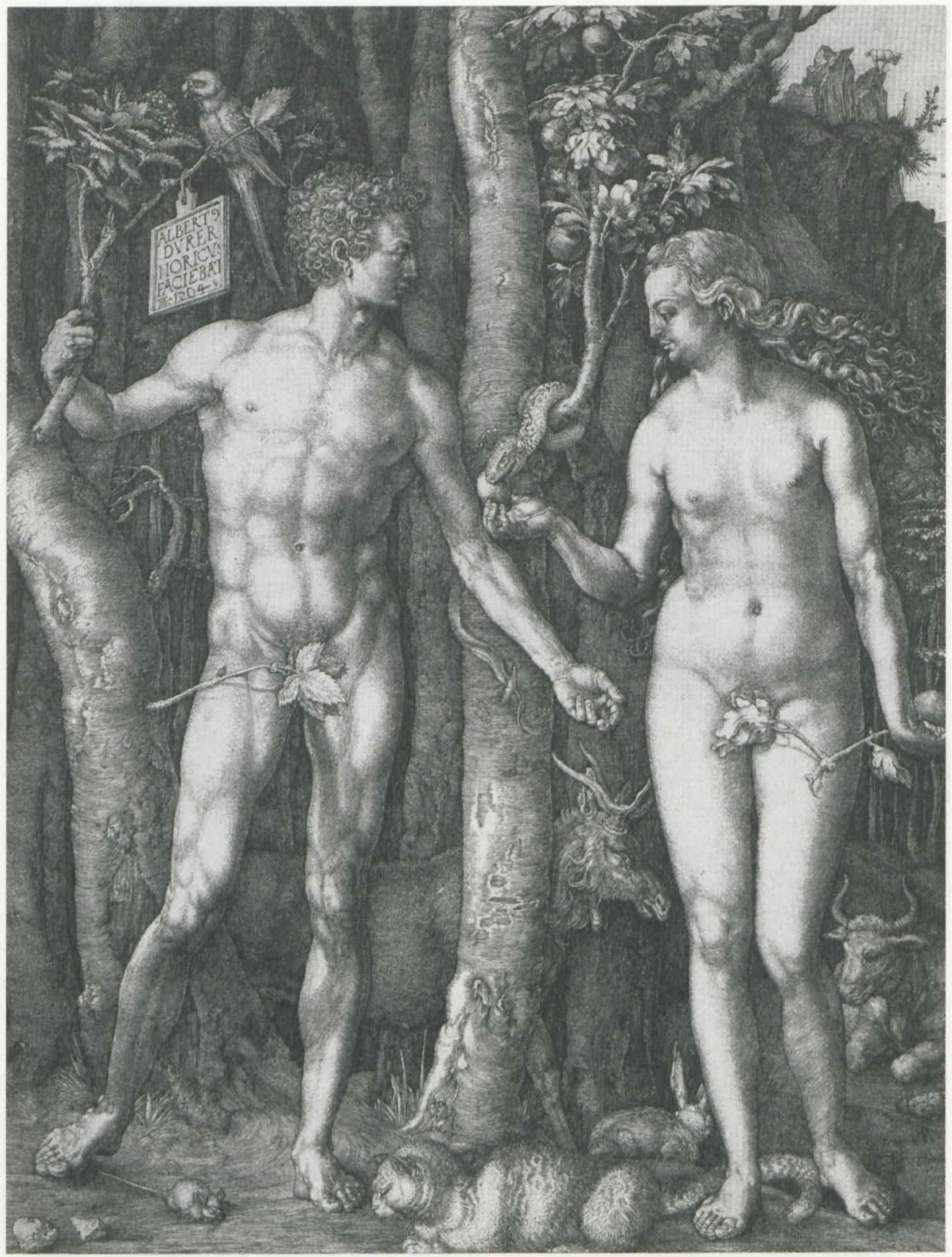

Abb. 3 Albrecht Dürer, Adam und Eva, Kupferstich, 1504 


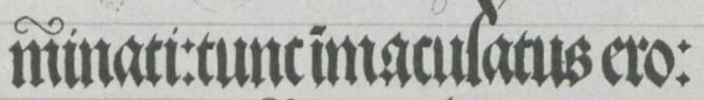
etemumbabiondsclitto matr mo.Etertunt pt complateceant cloquixo:sismettet mesoitatio co:sismeciintópipectutuoficm

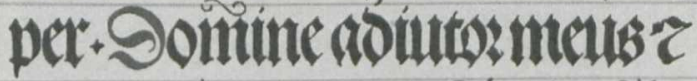

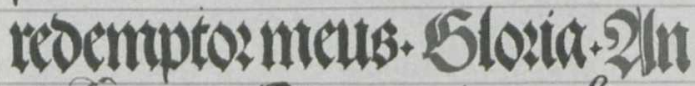
tiploonta. Sicut mutrta clectat

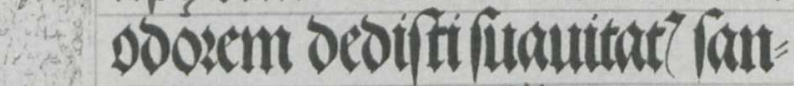

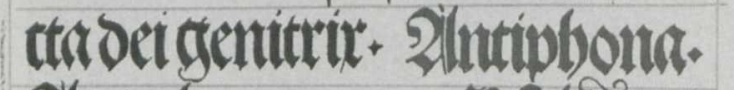

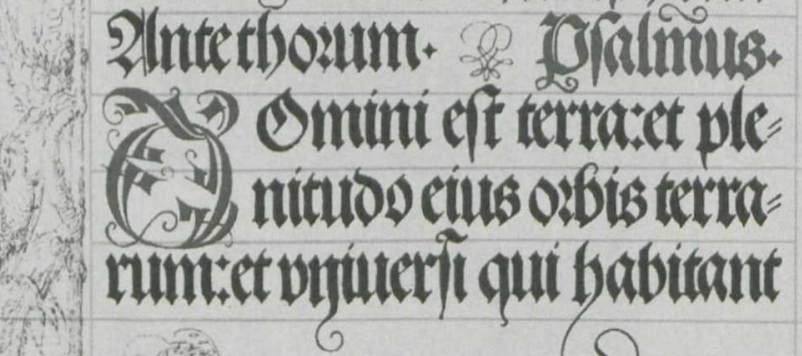

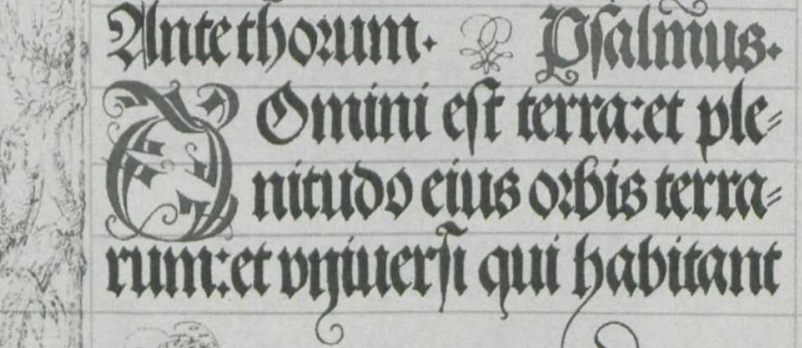

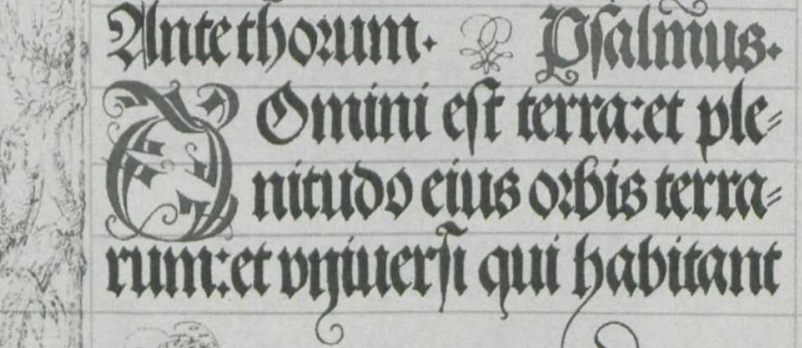

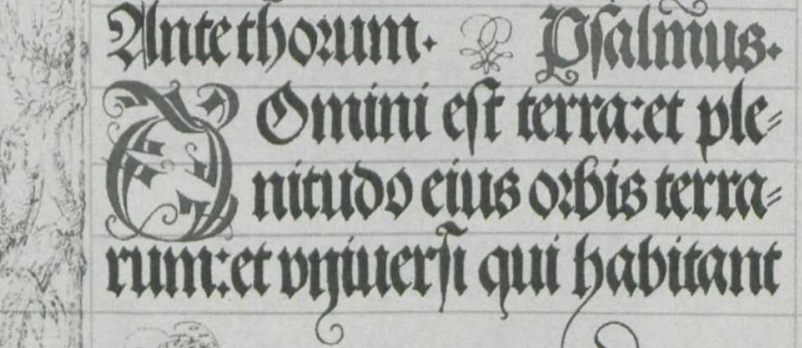
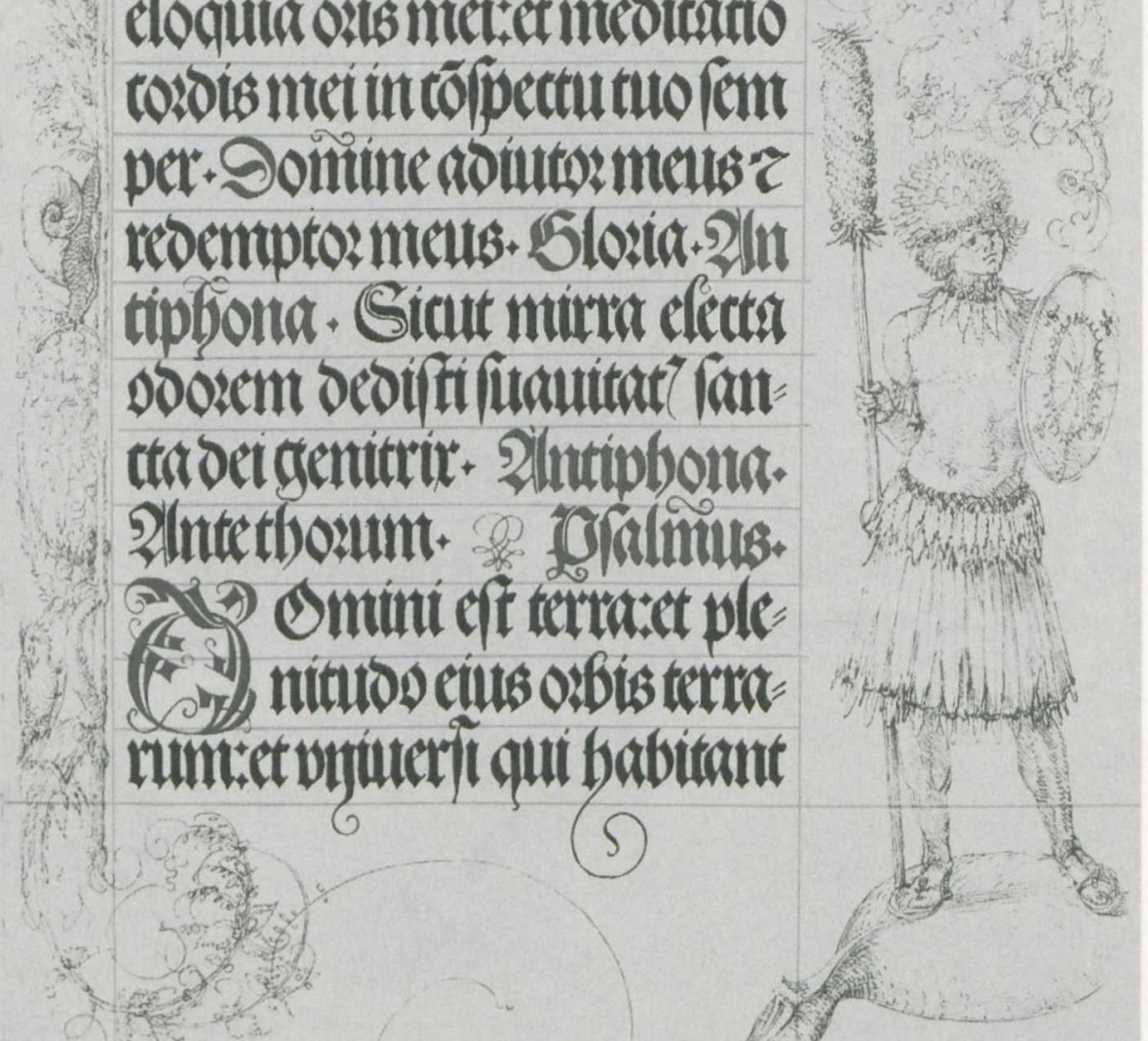

Abb. 4 München, BSB, $2^{\circ}$ L. impr. membr. $64, \mathrm{f}^{\circ} 41$.

Gebetbuch Kaiser Maximilians mit Darstellung eines „Indianers“ von Albrecht Dürer 


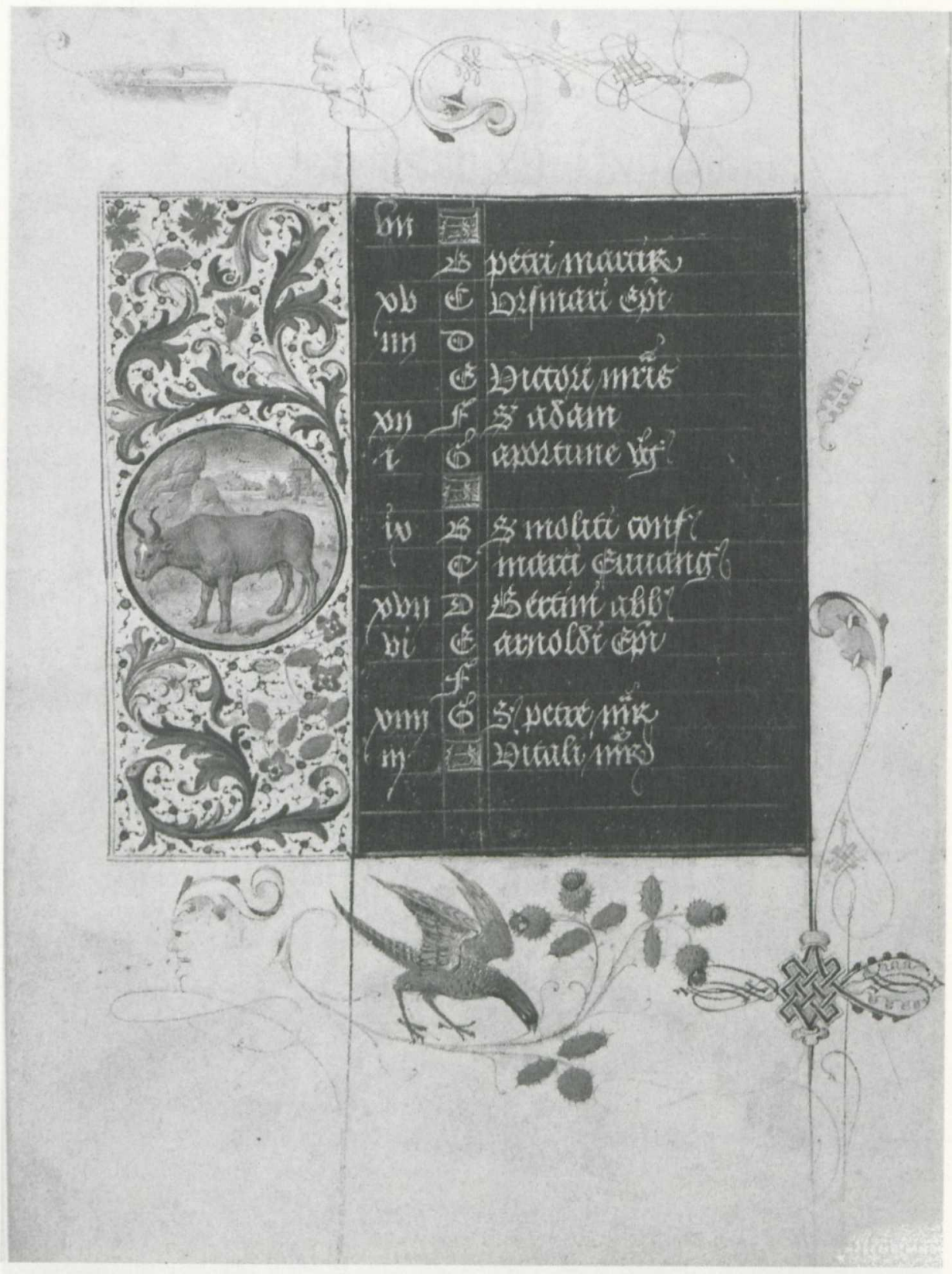

Abb. 5 Wien, Österreichische Nationalbibliothek, Cod. 1857, $\mathrm{f}^{\circ} 5 \mathrm{v}$ (Ausschnitt) 


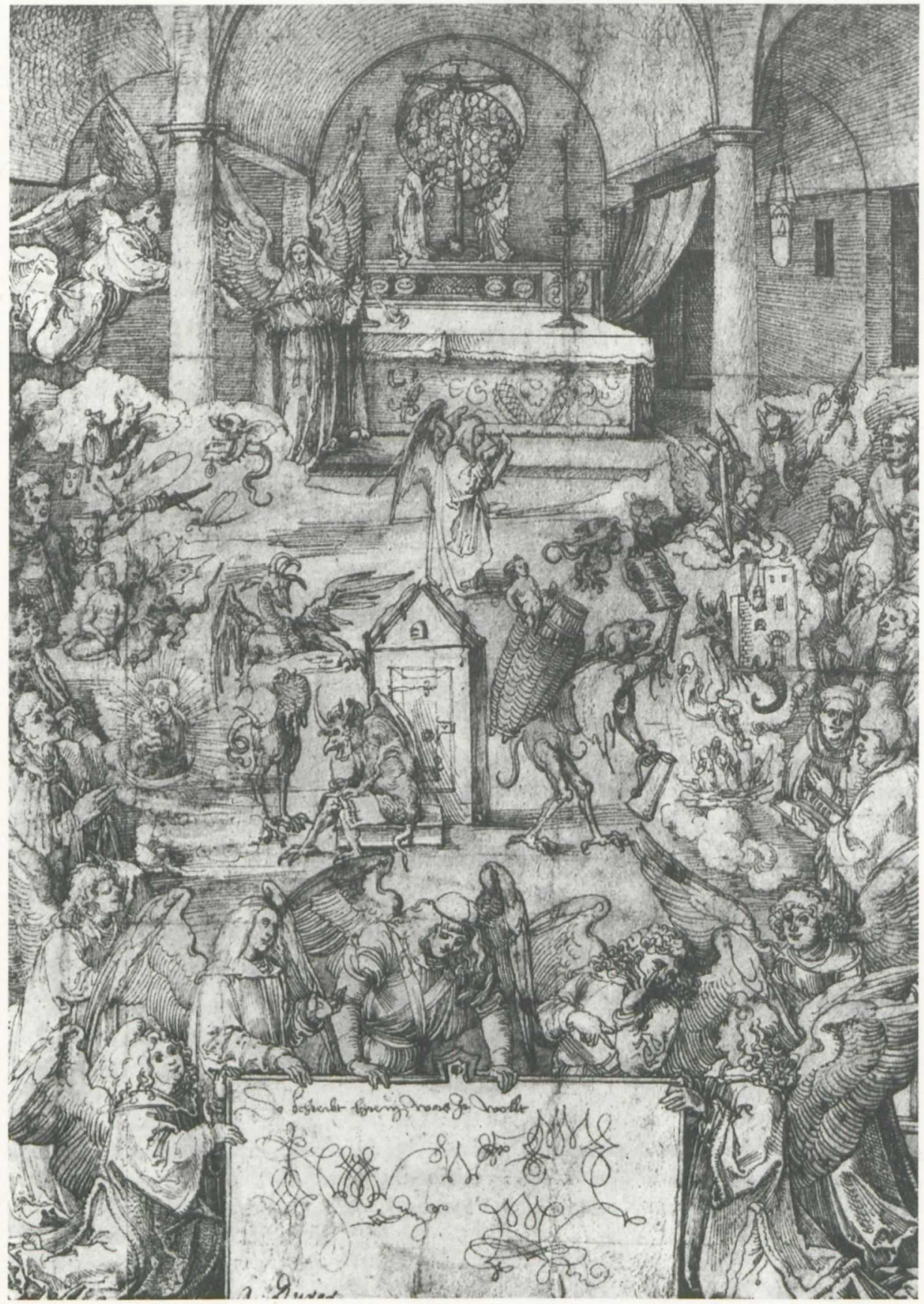

Abb. 6 Albrecht Dürer, sog. „Engelsmesse“ 